\title{
Zeta Functions: Symmetry and Applications +
}

\section{Emilio Elizalde}

National Higher Research Council of Spain, Institute for Space Sciences, UAB Campus, Carrer de Can Magrans s/n, 08193 Bellaterra (Barcelona), Spain; elizalde@ieec.uab.es

+ Presented at Symmetry 2017-The First International Conference on Symmetry, Barcelona, Spain, 16-18 October 2017.

Published: 3 January 2018

A short summary of some symmetry properties of the Riemann zeta function and of different zeta functions corresponding to pseudodifferential operators will be given. An introduction to the main issues concerning the zeta-function regularization procedure will follow, with special emphasis on the rigor, power, and universality of the method, contrasting with the sloppy missuses of the same that have appeared too often in the literature. After that, applications of zeta-function regularization in quantum field theory and cosmology will be discussed, together with some important associated problems that can be dealt with by using this method.

\section{References}

1. Elizalde, E. Ten Physical Applications of Spectral Zeta Functions, 2nd ed.; Lecture Notes in Physics; Springer-Verlag: Berlin, Germany, 2012.

2. Elizalde, E. Ten Physical Applications of Spectral Zeta Functions, 1st ed.; Lecture Notes in Physics Monographs; Springer-Verlag: Berlin, Germany, 1995.

3. Elizalde, E.; Odintsov, S.D.; Romeo, A.; Bytsenko, A.A.; Zerbini, S. Zeta Regularization Techniques with Applications; World Scientific: Singapore, 1994.

4. Kirsten, K. Spectral Functions in Mathematics and Physics, 1st ed.; Chapman \& Hall: London, UK, 2001.

5. Bytsenko, A.A.; Cognola, G.; Elizalde, E.; Moretti, V.; Zerbini, S. Analytic Aspects of Quantum Fields; World Scientific: Singapore, 2004.

(C) 2018 by the author. Licensee MDPI, Basel, Switzerland. This article is an open access article distributed under the terms and conditions of the Creative Commons Attribution (CC BY) license (http://creativecommons.org/licenses/by/4.0/). 